\title{
Método computacional para el cálculo de la eficiencia de perforación de pozos petrolíferos mediante el método de los elementos finitos
}

pags 111-121

Grupo de Investigación: Grupo de diseño avanzado y Nuevas tecnologias de Perforación Línea de investigación: Modelado Computacional y Nuevas tecnologías de perforación

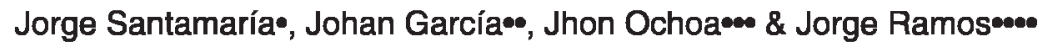

\section{RESUMEN}

En este artículo se exponen los resultados obtenidos mediante una metodología para calcular la relación entre la energía mecánica específica en superficie (MSEs) con la energía mecánica específica en fondo (MSEf) mediante el método de elementos finitos. Se utilizó un modelo CAD de una broca PDC y un modelo de roca homogénea, lineal e isotrópica. Fueron considerados datos de perforación que sugerían fenómenos vibratorios.

Se utilizó la energía de deformación como magnitud análoga a la MSE y se concluyó que la variación entre MSEs y MSEf se ve reflejada en la deformación adicional en los cortadores y cuerpo de la broca. Se halló que la relación entre la energía MSEs y MSEf es de tipo lineal directa bajo las condiciones de contorno asumidas. Finalmente se evaluó la eficiencia mecánica en la perforación del campo de estudio.

Palabras Clave: Energía mecánica específica, eficiencia de perforación, método de los elementos finitos.

\begin{abstract}
In this paper the relationship between MSEs and MSEf calculated via Finite Element Method are presented. For these purpose we have used a $C A D$ model of PDC bit and a homogeneus, lineal and isotropic model for rock properties. Data of vibrations phenomenon were considered.

The strain energy was used like analogical magnitude to MSE. It was concluded that the difference between MSEs and MSEf it's reflected on the adicional strain in cutters and body of the bit. It was found that the relationship between MSEs and MSEf is straightforward linear. Finally the drilling efficency was evaluated for the oilfield of studied.
\end{abstract}

Key Words: Mechanical Specific Energy, Rock driIling efficiency, Finite element method.

\footnotetext{
- Estudiante programa de Ingeniería Mecánica Fundación Universidad de América.

- Estudiante programa de ingeniería de Petróleos Fundación Universidad de América.

... Ingeniero Mecánico, Master en ingeniería de materiales. Docente investigador. Fundación Universidad de América. jhon. ochoa@unvestigadores.uamerica.edu.co

•... Ingeniero de Petróleos, Master en ingeniería. Docente investigador. Fundación Universidad de América. jorge.ramos@ investigadores.uamerica.edu.co
} 


\section{INTRODUCCIÓN}

El proceso de perforación para extracción de hidrocarburos, es parte fundamental de una cadena productiva que va desde la fabricación de envases hasta la generación de energía. La eficiencia del proceso de perforación ha sido objeto de estudio desde hace más de cincuenta años. Los primeros trabajos relacionados al tema en discusión, analizan las fuentes de disipación de energía durante el corte de un volumen de roca, donde se concluye que la mayor parte de la energía suministrada se gasta en la deformación elástica de volúmenes de roca subyacentes al volumen que se quiere fragmentar (Simon, 1963). Por su parte (Teale, 1964), define la Energia Mecánica Específica (MSE') mediante la relación de parámetros de perforación medidos en superficie y que realizan trabajo sobre la broca. Específicamente relaciona el trabajo del peso sobre la broca $\left(W^{2} B^{2}\right.$ ) y el trabajo hecho torque aplicado con las propiedades mecánicas de un volumen de roca.

La metodología de propuesta por (Teale, 1964), puede ser utilizada para cuantificar de la energía mecánica específica en superficie y también para su medición en fondo. La primera corresponde a la MSEs y se calcula a partir de los parámetros de perforación censados en superficie, lo que es equivalente al trabajo bruto suministrado durante la operación. La segunda corresponde a la MSEf y se cuantifica con los parámetros censados en fondo, y que equivale al trabajo neto que está siendo entregado a la broca. Existe una diferencia entre la MSEs y la MSEf debido a pérdidas energéticas propias del proceso y desempeño del ensamble que hay desde la superficie hasta el punto de perforación. Un estudio realizado en el golfo de México, (Thomson \& Mathur, 2010), reveló que la causa principal de la diferencia entre estas dos mediciones se debe principalmente a la aparición de fenómenos vibratorios en

1 Siglas en inglés de Mechanic Specific Energy

2 Siglas en inglés de Weight On Bit durante la operación de perforación. Los fenómenos vibratorios son susceptibles de análisis pues entran en la clasificación de las ineficiencias específicas definidas en (Pessier \& Fear, 1992). En esta investigación los fenómenos vibratorios de torsión fueron consideradas en un análisis de MSE general.

En el presente artículo se cuantificó la eficiencia de perforación de un pozo petrolífero, determinando la relación existente entre la MSEs y la MSEf. La primera cuantificada a partir de parámetros tomados en superficie, mientras que la segunda se calculó de manera aproximada mediante el método de los elementos finitos debido a la dificultad de su medición a partir de parámetros en fondo. Para el cálculo de la MSEf se consideró fenómenos de vibración torsionales a partir de una metodología propuesta aquí propuesta.

Los resultados de la investigación validan la hipótesis de que existe una disminución de energía mecánica específica en fondo (respecto a la de superficie) debido a las vibraciones torsionales. La importancia de esta investigación radica en que debido a la reciente implementación del parámetro de energía mecánica específica en Colombia y a la imposibilidad de medir parámetros en fondo, este tipo de modelos computacionales ofrecen una forma de cálculo aproximado de la eficiencia de perforación de una determinada sección sin necesidad de realizar mediciones de fondo en Campo.

\section{MATERIALES Y MÉTODOS}

A continuación se describe el procedimiento realizado para el cálculo de la relación entre MSEf y MSEs. Primero se presentan las definiciones propias de los conceptos utilizados en el cálculo realizado. Después se caracteriza la sección del pozo perforado objeto de estudio Al final de la sección se presenta la metodología de cálculo de la eficiencia de perforación. 
LÍNEA DE INVESTIGACIÓN: MODELADO COMPUTACIONAL Y NUEVAS TECNOLOGÍAS DE PERFORACIÓN

\subsection{Definiciones Teóricas}

Energía mecánica especifica (MSE): Es definida en (Teale, 1964) como la energía mecánica necesaria para fracturar un volumen de roca. Por su parte (Dupriest \& Koederitz, 2005), definen la ecuación 1 , modificando la ecuación original presentada por (Teale, 1964) donde relaciona los parámetros de perforación que realizan trabajo mecánico en la roca.

\section{Ecuación 1.}

$$
M S E[k s i]=\frac{W O B}{A_{b}}+\frac{120 \pi T R P M}{R O P A_{b}}
$$

Donde

WOB $=$ Peso sobre la broca [lbs]

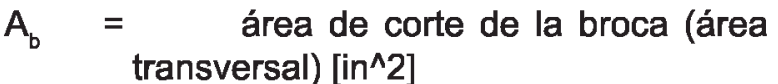

$\mathrm{T}=\quad$ Torque aplicado $[\mathrm{lb}-\mathrm{ft}]$

$\mathrm{RPM}=\quad$ velocidad de rotación [RPM]

ROP $=\quad$ tasa de penetración

Eficiencia de perforación: La eficiencia de la perforación se puede evaluar mediante la comparación de la MSE con la resistencia que ofrece la roca a ser cortada. Esta resistencia puede ser en condiciones atmosféricas, caso en el cual se denomina resistencia a la compresión de la roca sin confinar (UCS ${ }^{3}$ ) o en condiciones de presión diferentes a la atmosférica, denominándola resistencia a la compresión confinada $\left(\mathrm{CCS}^{4}\right)$. (Teale, 1964) Definió la energía mecánica específica mínima como aquella que se alcanza cuando la energía se aproxima a la resistencia a la compresión de la roca. Bajo éste concepto, la eficiencia máxima de perforación se obtiene al hacer el cociente entre la UCS MSE, como se muestra en la ecuación 2. La eficiencia máxima se tendría cuando el cociente es igual a 1.

3 Siglas de Unconfined Compressive Strength

4 Siglas de Confined Compressive Strength

$$
E f f=\frac{U C S}{M S E}
$$

\subsection{Generalidades del Pozo de Estudio}

El estudio se basó en parámetros de perforación del Campo Casabe; éste se encuentra ubicado a $350 \mathrm{~km}$ del norte de Bogotá en la Cuenca del Valle Medio del Magdalena, en el municipio de Yóndo Antioquia (Marquez, Elphick, Peralta , Amaya, \& Lozano , 2009). El campo fue seleccionado ya que allí se implementó la medición de la energía mecánica específica como método de optimización para la operación de perforación con los pozos piloto CB-1402, CB1419D Y CB- 1456. Adicionalmente se contaba con información de la dinámica de perforación en fondo de pozo de los pozos CB-1187 Y CB 1491 medida con sensores ubicados en la sarta de perforación y cercanos a la broca. Los datos considerados recopilan información de fenómenos vibratorios, de MSE y velocidad de rotación, además de la medición de parámetros de perforación en superficie.

Para el estudio se consideraron las secciones de perforación verticales del campo. Las propiedades geomecánicas de la Formación Mugrosa del Campo Casabe fueron consideradas mediante un análisis estadístico, ya que en esta formación hubo un aumento de MSEs y vibraciones en fondo para los pozos ubicados en el bloque 1 y 3 del campo mencionado. En el análisis se escogió un intervalo de $4800 \mathrm{ft} \mathrm{a}$ $6700 \mathrm{ft}$ correspondiente a la formación de estudio, en donde se consideraron los cuatro tipos de roca existentes, arenisca (Vsh<0.3), limolita $(0.3<$ Vsh $<0.5)$, arcillolita $(0.5<$ Vsh $<0.8)$ y arcilla (Vsh>0.8). En las Tablas 1-2 se presentan las propiedades promedio de las rocas predominantes en esta formación. El análisis estadístico realizado permitió identificar el tipo de roca y propiedades mecánicas predominante en la Formación de interés. La formación rocosa se consideró como un sólido homogéneo, elástico lineal e isotrópico. 
Tabla 1. Propiedades geomecánicas a un volumen de arcilla (Vsh) menor a $\mathbf{0 . 3}$

\begin{tabular}{|l|c|c|c|c|}
\hline \multicolumn{5}{|c|}{ Propiedades promedio 4800' - 6700' } \\
Propledad & Promedio & Minimo & Maximo & Desvlación estandar \\
\hline GR (api) & 61,3101718 & 39,9671 & 69,99634 & 6,25916093 \\
\hline Vsh & 0,21310194 & 0 & 0,2999634 & 0,06259085 \\
\hline RHOB (g/cc) & 2,38655764 & 2,23927 & 2,56024 & 0,05011491 \\
\hline PHIT & 0,15988913 & 0,08649 & 0,23352 & 0,02564007 \\
\hline Poisson & 0,32520061 & 0,42787893 & 0,42787893 & 0,0313171 \\
\hline Young E (psi) & 2930075,61 & 1479500,48 & 4811576,43 & 518475,819 \\
\hline UCS (PSI) & 4579,21056 & 2299,80612 & 9101,01867 & 1268,22703 \\
\hline No. datos / propiedad & 1471 & \multicolumn{4}{|l}{} \\
\hline
\end{tabular}

Fuente: los autores

Tabla 2. Propiedades geomecánicas a un volumen de arcilla (Vsh) entre 0.3 y 0.5

\begin{tabular}{|l|c|c|c|c|}
\hline \multicolumn{5}{|c|}{ Propiedades promedio 4800' - 6700' } \\
\hline Propiedad & Promedio & Minimo & Máximo & Desviación estandar \\
\hline GR (Api) & 79,9794872 & 70,00309 & 89,97784 & 5,53175611 \\
\hline Vsh & 0,39979487 & 0,3000309 & 0,4997784 & 0,05531756 \\
\hline RHOB (g/cm3) & 2,41299679 & 2,16226 & 2,59425 & 0,06128693 \\
\hline PHIT & 0,15095658 & 0,07635 & 0,27272 & 0,02958047 \\
\hline Poisson & 0,35905935 & 0,24340961 & 0,44625514 & 0,04050833 \\
\hline Young E (PSI) & 2633299,95 & 933613,324 & 4899765,43 & 727111,496 \\
\hline UCS (PSI) & 5211,16292 & 1510,45745 & 10032,4976 & 1477,45442 \\
\hline No. datos / propiedad & 2503 & & & \\
\hline
\end{tabular}

Fuente: los autores

Se analizaron parámetros de perforación y MSE de superficie en 7 pozos del Campo Casabe, tres de ellos ubicados en el bloque $1 \mathrm{y}$ cuatro ubicados en el bloque 3 de los cuales dos tenían medición de vibraciones en fondo pozo. A dichos pozos se les realizó un análisis por formación donde se determinaron sus valores promedio correspondientes de los parámetros de perforación y MSE de superficie.

De igual manera se analizaron los pozos que contenían información de vibraciones donde se obtuvo valores promedio de aceleraciones centrípetas que variaron de $2 \mathrm{~g}$ a $3 \mathrm{~g}$, aceleraciones laterales de $0.85 \mathrm{~g}$ a $0.90 \mathrm{~g}$ y valores máximos que variaron entre $5 \mathrm{~g}$ a $7 \mathrm{~g}$, siendo los valores de aceleración más altos en la Formación Mugrosa.

En la Figura 1 se observan incrementos de la energía mecánica específica. Éstos se pre- sentan cuando hay dificultad para perforar, lo que obedece a una disminución de la ROP. Para continuar con la perforación es deben aumentar los requerimientos de peso sobre la broca y torque, pues solo así se logra vencer el torque reactivo generado.

En la Figura 2 se pude observar la influencia de las vibraciones torsionales en la energía mecánica específica de superficie, donde se evidencia que al aumentar las vibraciones torsionales en fondo de pozo aumenta la el valor de la MSE, tal como lo descrito por $\mathbf{R}$. PESSIER, Ref. 7, en donde los aumentos de MSE son generados por aumentos de torque y arrastre, además de la aparición del fenómeno del stick-slip dando un indicador de que existe alguna restricción al momento de perforar la cual genera una ineficiencia en dicho proceso. 
LÍNEA DE INVESTIGACIÓN: MODELADO COMPUTACIONAL Y NUEVAS TECNOLOGÍAS DE PERFORACIÓN

\section{Gráfica 1. Comportamiento de MSE en el Campo Casabe}

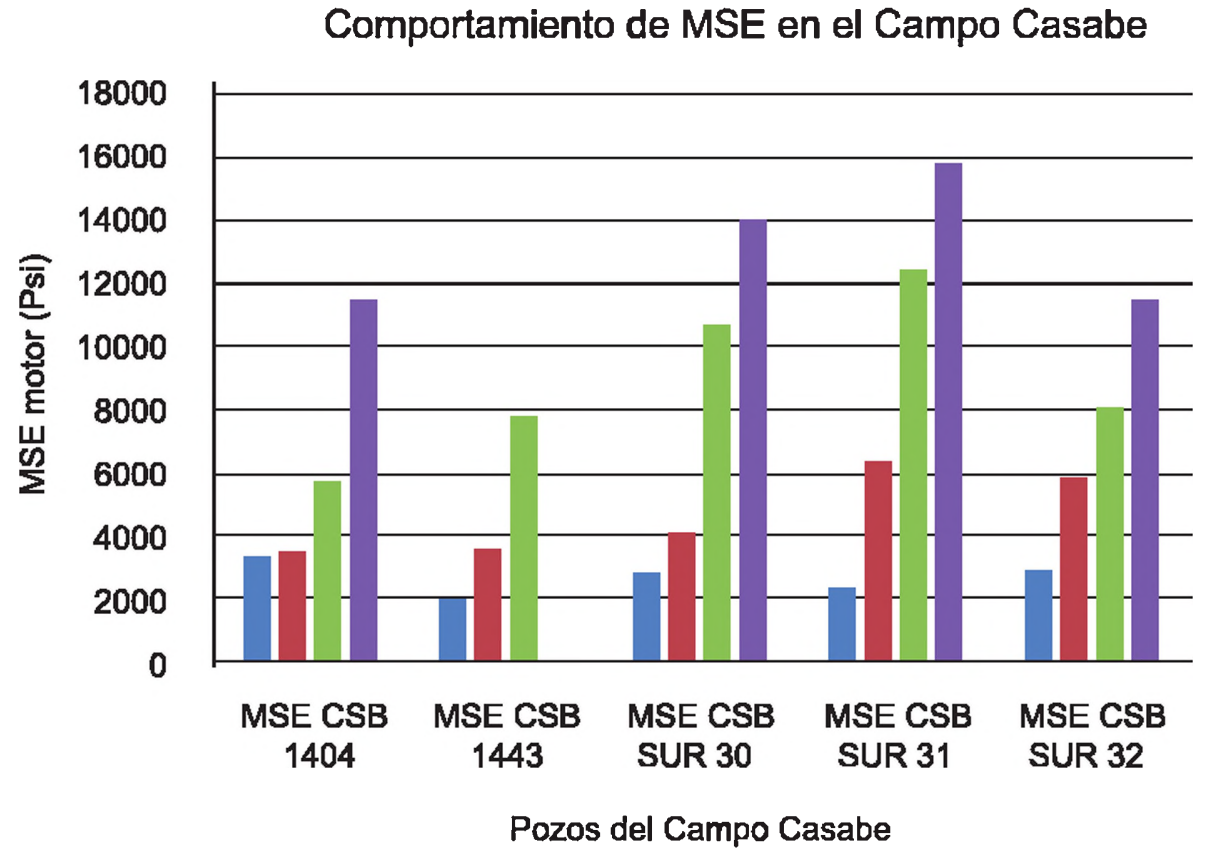

Fuente: los autores

Figura 1 Registro Masterlog de la Formación Mugrosa del pozo Casabe 1187.

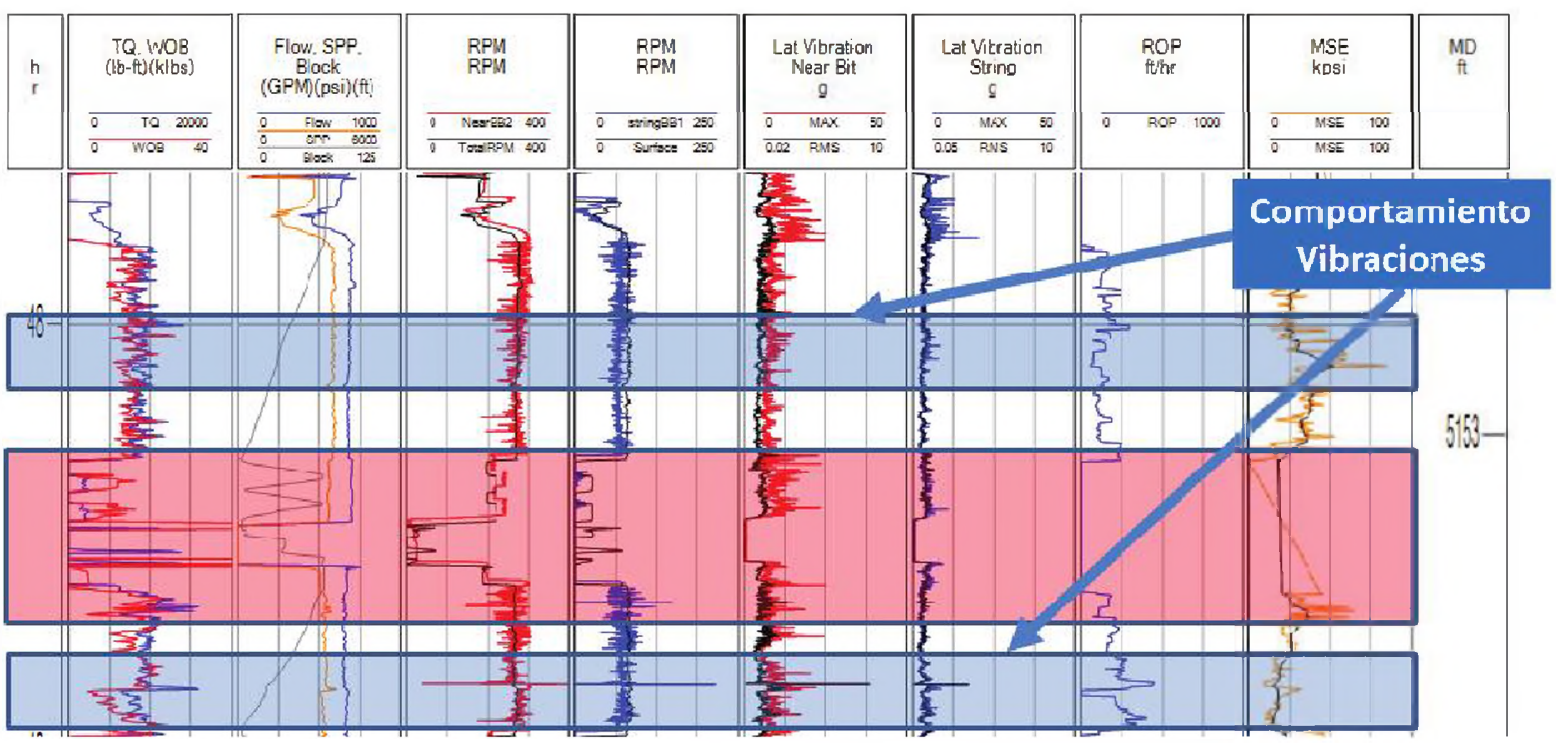

Fuente: (National Oilweel Varco - Integrated Solution and Optimization, Sección 8.5" x 9.5." p 29 .) 
Figura 2. MSE y vibraciones torsionales del pozo Casabe 1187 en la Formación Mugrosa
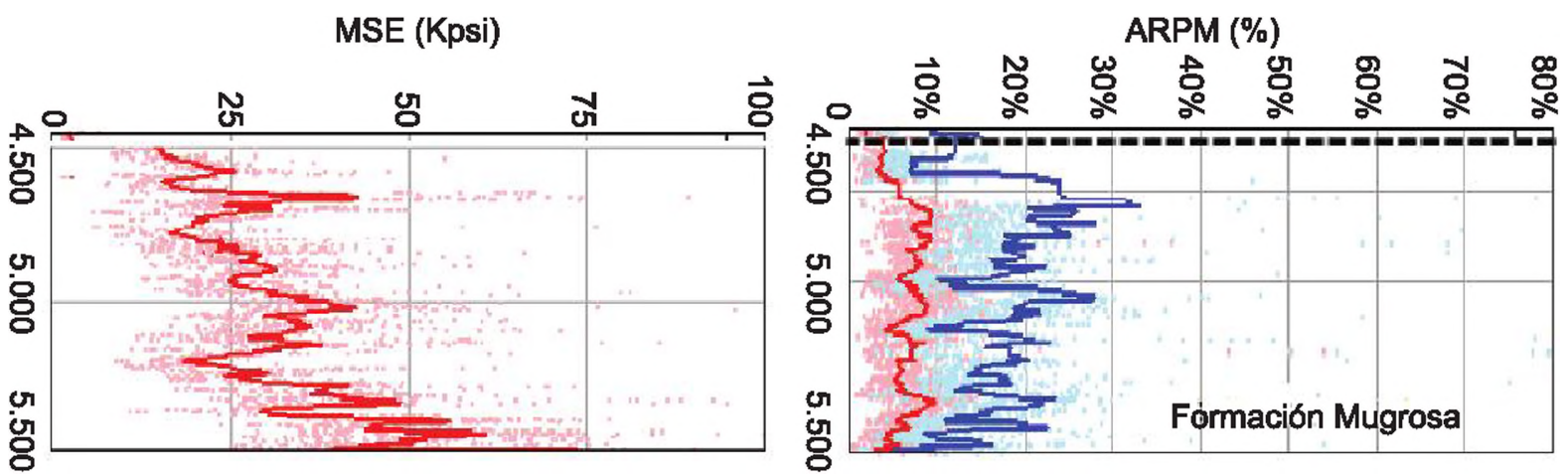

Fuente: (National Oilweel Varco - Integrated Solution and Optimization, Sección 8.5” x 9.5.p 29 .)

\subsection{Determinación aproximada de la MSE en Fondo}

Se realizaron simulaciones por medio del Software Ansys Workbench, donde se incluyó una carga de tipo axial que representó el peso sobre la broca, y un momento que hizo las veces de torque sobre la broca. La figura 3 muestra cómo se configuró la broca, de manera tal que cada cortador estaba en contacto con un bloque de formación rocosa. Toda la geometría propia de operación se estableció por medio del software mencionado.

Figura 3. Configuración broca PDC y bloques

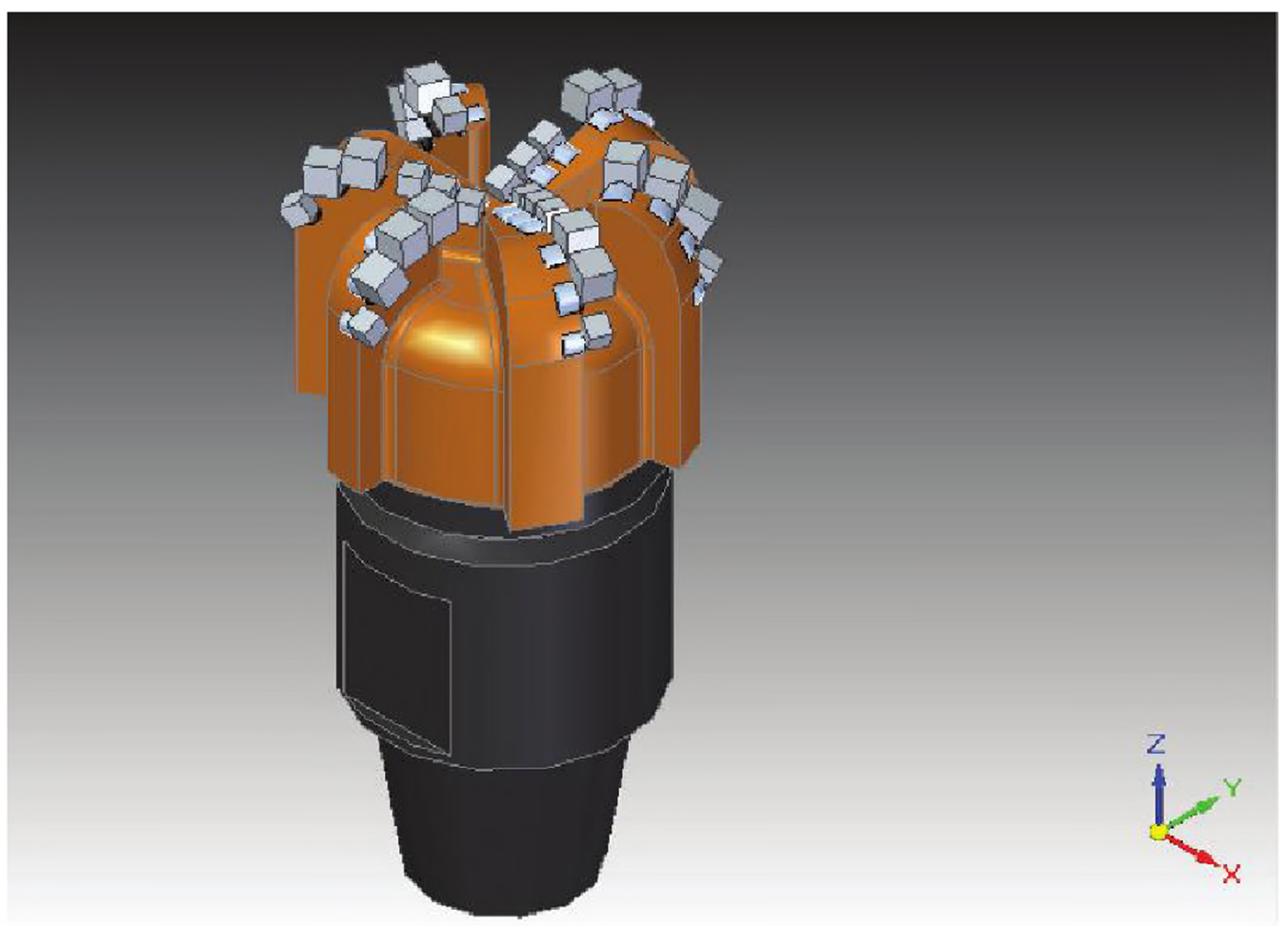

Fuente: los autores 
LINNEA DE INVESTIGACIÓN: MODELADO COMPUTACIONAL Y NUEVAS TECNOLOGÍAS DE PERFORACIÓN

En la figura 4 se muestra las restricciones mecánicas utilizadas en la simulación. La etiqueta " $A$ " representa la restricción propia del comportamiento normal de la broca, mientras que la etiqueta " $B$ " simboliza la restricción adicional generada por el atascamiento debido a vibraciones torsionales.

Figura 4. Condición de atascamiento de la broca PDC.

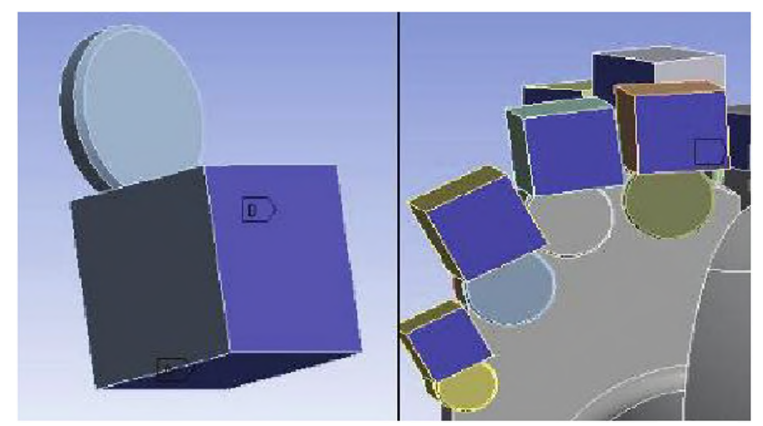

Fuente: los autores

El siguiente diagrama de flujo de la figura 5, muestra el proceso para la obtención de la MSE, tanto de superficie como de fondo. Es importante resaltar el uso de la energía de deformación como concepto análogo a la energía mecánica específica, esto basándose en lo concluido por (Simon, 1963). La simulación se detiene en $6.5 \mathrm{~mm}$ debido a que por la forma de corte de las brocas PDC, el cortador solo llega a introducirse hasta la mitad de su diámetro, esto sumado a que resulta dificil saber con exactitud cuánto se introduce cada cortador.

Tabla 3. Parámetros asociados al evento más severo de stick-slip

\begin{tabular}{|c|c|c|c|c|c|c|}
\hline $\begin{array}{l}\text { Conjunto de } \\
\text { partimetros }\end{array}$ & $\begin{array}{l}\text { Profundidad } \\
\text { de corte (mm) }\end{array}$ & ROP (tthr) & WOB (klbs) & Torque (lb-ft) & $\begin{array}{l}\text { RPH superficie } \\
\text { (Jpm) }\end{array}$ & $\begin{array}{c}\text { MSE } \\
\text { supericie (ksi) }\end{array}$ \\
\hline 1 & 2.075 & 313.65 & 19.1 & 12815.3 & 78 & 17.21955 \\
\hline 2 & 3.55 & 297.5 & 16 & 11948.3 & 76 & 16.45977 \\
\hline 3 & $5 . \overline{025}$ & 297.5 & $\overline{7.7}$ & 8960.24 & 84 & 13.56432 \\
\hline 4 & 6.5 & 297.5 & 3.8 & 9766.74 & 86 & 15.06964 \\
\hline Promedio & $\mathrm{n} / \mathrm{a}$ & 301.5375 & 11.65 & 10872.65 & 81 & 15.69799 \\
\hline
\end{tabular}

Fuente: los autores

5 Las vibraciones torsionales son conocidas como fenómeno stick-slip
Debido a la gran cantidad de simulaciones necesarias para una sola configuración de peso sobre la broca y torque, se decide adoptar la metodología planteada por (Zhou, y otros, 2012), en donde la energía mecánica especifica de superficie tiene un comportamiento de decaimiento exponencial y está en función de la profundidad de corte. De manera tal que una vez se ha alcanzado cierta profundidad se estabiliza. Este comportamiento se muestra en la gráfica 2.

Bajo éste comportamiento se procedieron a simular varios escenarios planteados tanto para MSEs como para MSEf. En la simulación se escogieron los parámetros registrados durante el evento de stick-clip más severo presentado durante la perforación del pozo CSB 1187, estos se muestran en la tabla 3. Cada conjunto de parámetros se aplicó hasta la profundidad de corte mostrada en la tabla 3, de manera que se plantearon tres escenarios, el primero con un $25 \%$ de restricción, es decir se aplicó la restricción adicional durante el conjunto de parámetros 1. El segundo con un $50 \%$ de restricción (restricción adicional para el conjunto de parámetros 1 y 2). $Y$ el tercero con $100 \%$ de restricción, lo que implica que la restricción adicional se aplicó durante toda la simulación. Es de resaltar que en los momentos en que tuvo lugar la restricción adicional, la energía de deformación se vio reflejada en deformación adicional de los cortadores de la broca, lo cual quiere decir que se empleó menos energía en "cortar" la roca. 


\section{Figura 5. Diagrama de flujo para el cálculo}

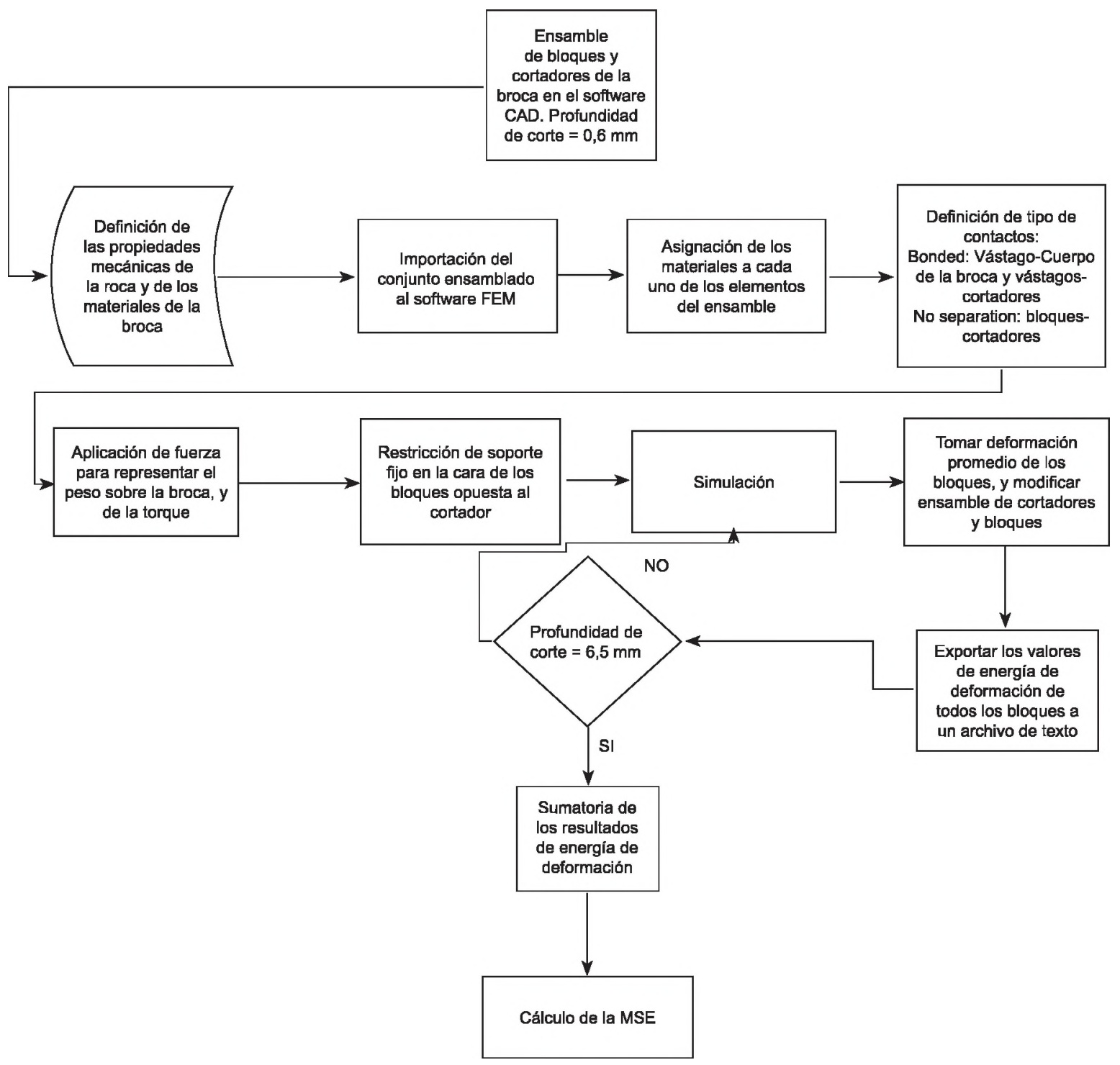

Fuente: los autores 
LÍNEA DE INVESTIGACIÓN: MODELADO COMPUTACIONAL Y NUEVAS TECNOLOGÍAS DE PERFORACIÓN

\section{Gráfica 2. MSEs vs profundidad de corte}

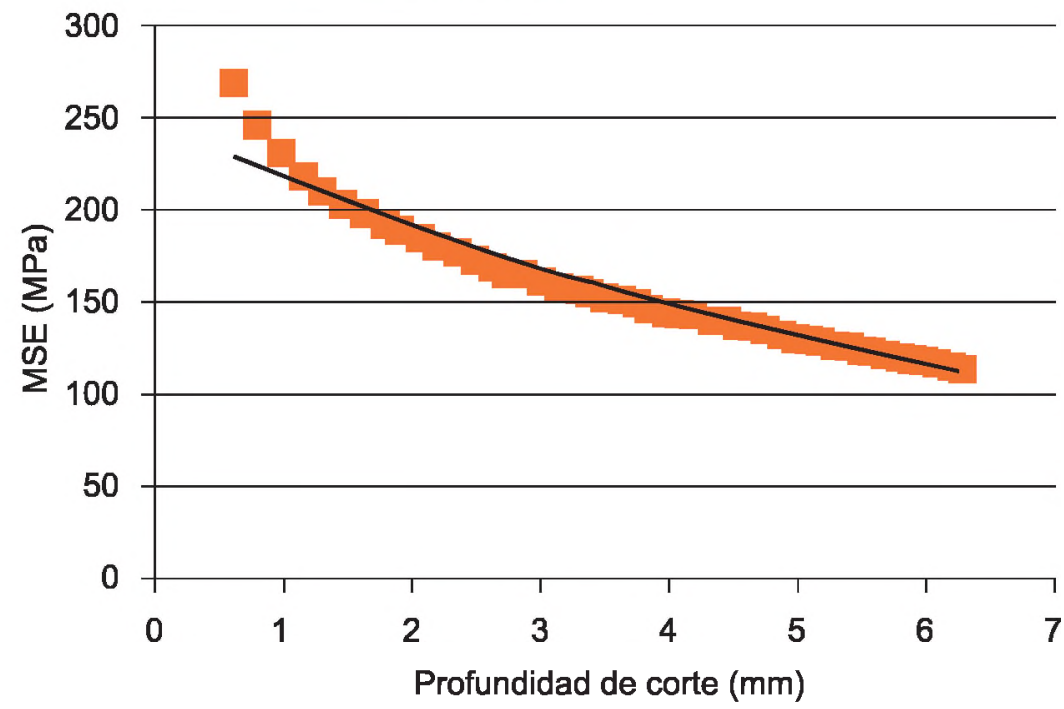

Parámetros:

WOB: $26,6 \mathrm{klb}$

Torque: $12767 \mathrm{lb}$-ft

Roca: Arenisca

MSE vs profundidad

Exponencial (MSE vs profundidad

$y=247,23 e^{-0,127 x}$

$R^{2}=0,9694$

Fuente: los autores

De los escenarios planteados el que mejor comportamiento presentó fue el que se simuló con un $50 \%$ de restricción adicional durante el evento de stick-slip, restringiendo el bloque para el conjunto de parámetros 1 y 2 , hasta una profundidad de $3.55 \mathrm{~mm}$, después se quitó dicha restricción y se aplicaron los parámetros 3 y 4 hasta la profundidad final.

\section{RESULTADOS}

La Gráfica 3 muestra las curvas de MSE en superficie (azul) y MSE en fondo (gris) obtenidas con la simulación. La zona azul representa una pérdida de energía e debida a una deformación a la deformación adicional de la broca. La curva de MSE de fondo sigue la tendencia de la MSE de superficie, aunque sin llegar a solaparla.

Gráfica 3. MSE (superficie y fondo) vs profundidad de corte para restricción del $50 \%$

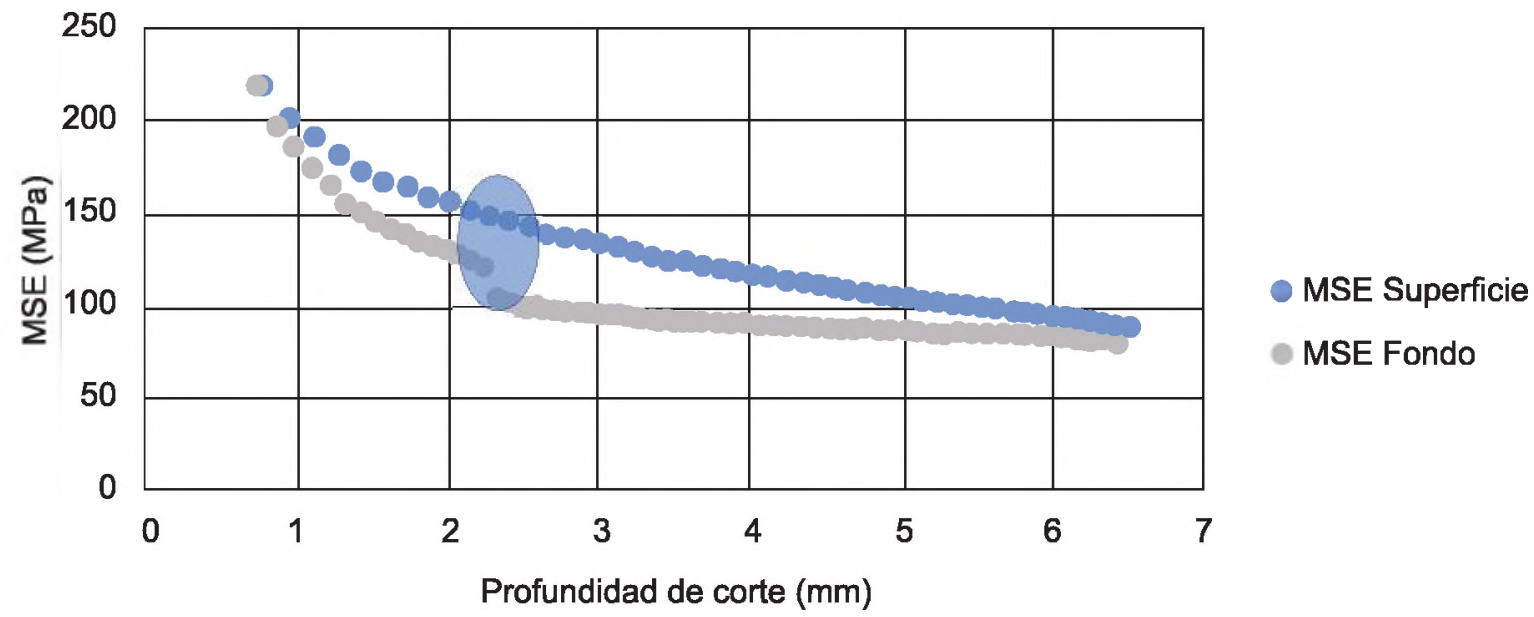

Fuente: los autores 
En la Gráfica 4 se muestra el comportamiento esperado entre la MSEs y la MSEf. Se observa que los valores son cercanos y el salto en la gráfica representa la pérdida de energía debida al evento de stick-slip. La Ecuación 3 muestra la recta que ajusta a la MSEf como función de la MSEs.

\section{Gráfica 4. MSEf vs MSEs para restricción del $\mathbf{5 0 \%}$}

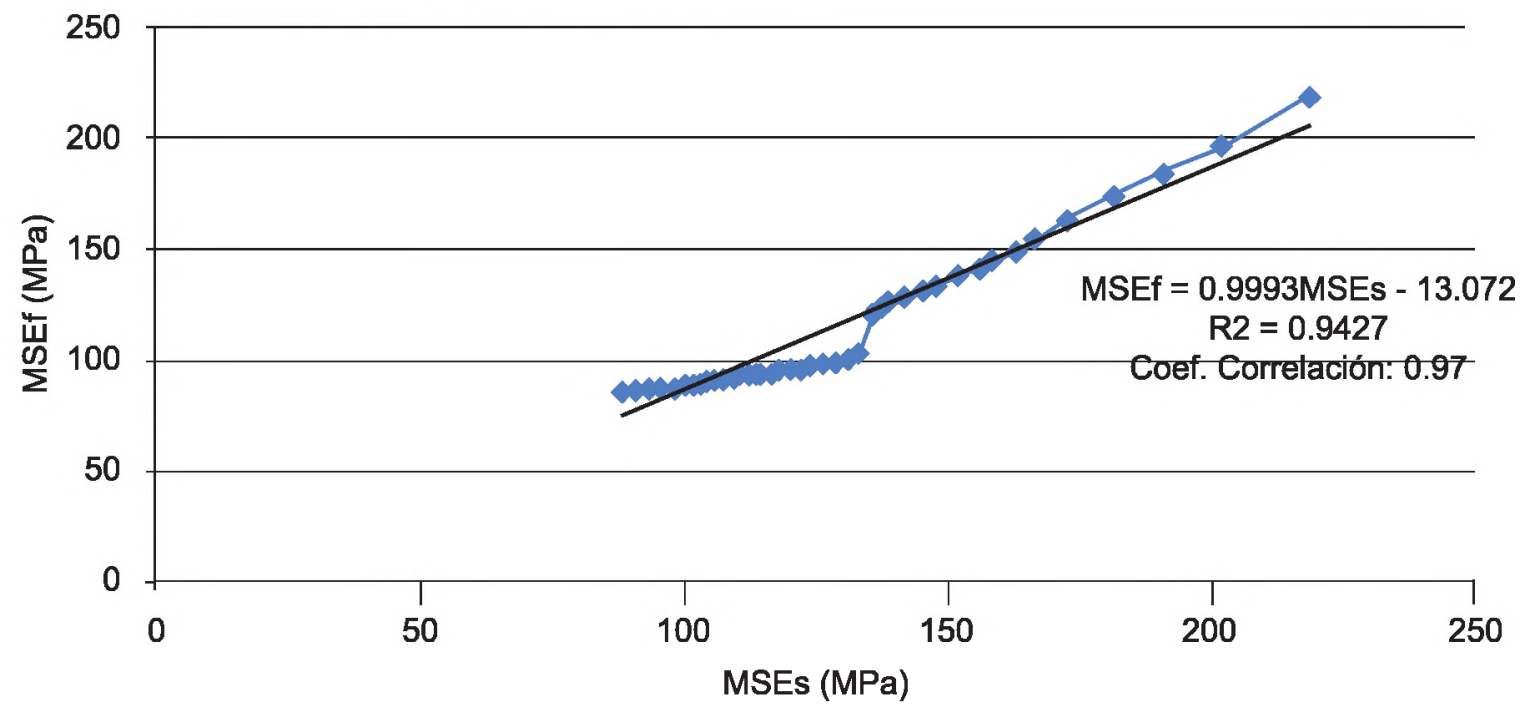

Fuente: los autores

Ecuación 3. Relación entre MSEf y MSEs para restricción del $50 \%$

$$
\mathrm{MSE}_{\mathrm{f}}=0.9993 \mathrm{MSE}_{\mathrm{s}}-13.072
$$

Para: 80MPa $\leq \mathrm{MSE} s \leq 218 \mathrm{MPa}$

\section{DISCUSÍON Y CONCLUSIONES}

Haciendo uso de simulaciones mediante el método de los elementos finitos, para modelar la interacción de una broca PDC con la roca, se logró validar la hipótesis planteada de que la pérdida de energía desde superficie hasta fondo se debe, en cierta medida, a eventos de vibraciones.

La relación entre la MSEs y la MSEf es de tipo lineal para el caso de estudio analizado y bajo las condiciones de contorno asumidas. Al graficar la MSEf en función de la MSEs se observan saltos en la recta que se deben a la pérdida de energía. Adicionalmente se valida el uso de la energía de deformación como magni- tud análoga a la MSE (tanto de superficie como de fondo) en la metodología planteada.

El uso de un modelo estático limita el estudio, de manera que la energía de deformación solo se ve afectada por el peso sobre la broca $y$ el torque, sin embargo se utilizó esta metodología para calcular la MSEs medida en pozo. El modelo presentado permite calcular la MSEs con errores relativos de entre el $2 \%$ y el $15 \%$ con respecto a los repostados en el historial de perforación del pozo de estudio.

Se sugiere validar el modelo anteriormente presentado mediante corridas de perforación en campo con parámetros similares y con la instrumentación necesaria para tomar datos de fondo, finalmente vale la pena resaltar que los efectos debido al resto del ensamblaje de fondo, el fluido de perforación y la tubería de perforación podrían cambiar significativamente los valores obtenidos ya que el modelo aquí presentado es estático-lineal y no contempla las no linealidades presentes en un proceso de perforación. 
LIINEA DE INVESTIGACIÓN: MODELADO COMPUTACIONAL Y NUEVAS TECNOLOGIAS DE PERFORACIÓN

\section{REFERENCIAS}

Dupriest, F. E., \& Koederitz, W. L. (2005). Maximizing Drill Rates with Real-Time Surveillance of Mechanical Specific Energy. SPE/IADC Drilling Conference. Amsterdam: Society of Petroleum Engineers.

Marquez, I. J., Elphick, J. J., Peralta , J. D., Amaya, M., \& Lozano , E. (2009). Casabe Mature Field Revitalization Throuhg an Alliance: A Case Study of Multicompany and Multidiciplinary Integration. Latin America and Caribbean Petroleum Engineering Conference. Cartagena de Indias, Colombia: Society of Petroleum Engineers.

National Oilweel Varco - Integrated Solution and Optimi- zation. (Sección 8.5" x 9.5". p 29 .). Casabe 1187.

Pessier, R. C., \& Fear, M. J. (1992). Quantifying Common Drilling Problems With Mechanical Specific Energy and BitSpecific Coefficeint of Sliding Friction. SPE Annual Technical Conference and Exhibition. Washington D.C.: Society of Pretroleum Engineers.

Simon, R. (1963). Energy Balance in Rock Drilling. Soiecty of Petroleum Egineers Journal, 3, 289-306.

Teale, R. (1964). The Concept of Especific Energy in Rock Drilling. International Journal of rock Mechanics and Mining Sciences, 2, 57-73.
Thomson, I. J., \& Mathur, R. (2010). The Use of Downhole Drilling Parameters Combined With Surface and Downhole Mechanical Specific Energy Data helped Identify Underreamer Dysfunctios In GoM Deepwater Projects. SPE Deepwater Drilling and Completions Conference. Galsveston, Texas: Society of Petroleum Engineers.

Zhou, Y., Zhang, W., Gamwo, I. K., Lin, J. S., Eastman, H., Whipple, G., \& Gill, M. (2012). Mechanical Specific Energy Versus Depth of Cut. 46th U.S. Rock Mechanics/ Geomechanics Symposium. Chicago, Illinois: American Rock Mechanics Associataion. 